\title{
Novel Nanostructures for Efficient Photon Upconversion and High-efficiency Photovoltaics
}

\author{
Diane G. Sellers ${ }^{\mathrm{a}}$, Jing Zhang ${ }^{\mathrm{a}}$, Eric Y. Chen ${ }^{\mathrm{a}}$, Yujun Zhong ${ }^{\mathrm{a}}$, Matthew F. \\ Doty $^{a, *}$, Joshua M. O. Zide ${ }^{\mathrm{a}}$ \\ ${ }^{a}$ Dept. of Materials Science and Engineering, University of Delaware, Newark, DE 19716
}

\begin{abstract}
Upconversion of low-energy photons theoretically allows the creation of singlejunction solar cells with efficiency far above the Shockley-Queisser (SQ) limit. However, the net efficiency gains that can be realized depend critically on details of the upconversion process employed. We define three important metrics of the performance of an upconversion material: upconversion quantum efficiency (UQE), photon energy sacrifice (PES), and absorption bandwidth (AB). We analyze the performance of existing upconversion materials relative to both these metrics and existing computational models of a single-junction photovoltaic (PV) cell backed by an upconverter. Guided by the results of this analysis, we develop a design for new solid state upconversion nanostructures that suppresses the dominant energy loss pathways and can enable substantial improvements in overall solar energy harvesting. We describe and model the performance of a specific realization of this design that uses an InAs quantum dot (QD) and a graded InAlBiAs layer to suppress both radiative and nonradiative loss pathways. We show that this design can be tailored to maximize upconversion efficiency and can enable a practical upconversion-backed PV system to exceed the SQ limit.

Keywords: detailed balance, dilute bismuthides, photovoltaics, quantum dot, upconversion
\end{abstract}

${ }^{*}$ Corresponding author

**doty@udel.edu

Preprint submitted to Solar Energy Materials and Solar Cells

May 23, 2016

(C) 2016. This manuscript version is made available under the Elsevier user license http://www.elsevier.com/open-access/userlicense/1.0/ 


\section{Introduction}

Solar cells are a promising path towards the development of renewable energy technologies that can displace fossil fuels, but many challenges must be overcome. A key challenge is the reduction of the installed cost per watt, which can be lowered by reducing the installed module cost or increasing the power generated per module. Research on first and second generation solar cell technologies will lead to increases in practical efficiency and decreases in module costs, but the theoretical maximum solar energy conversion efficiency of $33 \%$ places a limit on the total reduction in balance of systems costs that can be realized with these approaches. [1] The primary origins of the $33 \%$ limit derived by Shockley and Queisser for a single-junction photovoltaic (PV) are: 1) photons with energy larger than the bandgap lose their excess energy to heat, 2) photons with energy below the bandgap cannot be absorbed, 3) the formation of p-n junctions sacrifices energy to guide carrier transport and allow photocurrent to be harvested, and 4) a p-n junction at equilibrium has equal optical absorption and recombination and the power output is zero. Third generation solar cells utilize the solar spectrum in ways that overcome the Shockley-Queisser (SQ) limit. For example, multiple exciton generation (MEG) PV devices convert photons with energies at least twice the bandgap into two electron-hole pairs, thus doubling the photocurrent generated by these high-energy photons. There has been extensive work to produce MEG solar cells, but simultaneously enhancing MEG and extracting photocurrent is extremely challenging and the efficiency of existing devices remains poor. 2, 3] Multi-junction solar cells, which utilize multiple materials with different bandgaps to absorb sunlight over the whole spectrum, have demonstrated efficiency in excess of $40 \%$ using threejunctions based on III-V semiconductors. 4, 5, 6] However, the material growth cost and current matching requirements continue to be a significant challenge for cost-effective multi-junction solar cells. Additionally, the need for current matching makes multijunction devices extremely susceptible to changes in the solar spectrum (e.g. clouds), which can result in practical efficiencies below that 
of the single-junction cell. Intermediate band solar cells (IBSC) introduce energy levels in the middle of the bandgap to improve overall efficiency by enabling absorption of a wider portion of the solar spectrum. 7, 8, Although intermediate bands within wide bandgap materials have been realized with quantum dots, [9] band anticrossing, [10] and impurity levels, [11] the additional loss pathways introduced by the intermediate levels have outweighed any efficiency gains from utilizing low-energy photons. 12 .

Photon upconversion is another approach that utilizes more of the solar spectrum in order to improve overall solar energy conversion efficiency.[13, 14, 15. Upconversion-based PV is conceptually simple. High-energy photons are harvested by a host PV cell. Photons with energy below the host cell bandgap pass through the host cell to an upconversion material. Within the upconversion material sequential absorption of two or more low-energy photons promotes an electron through intermediate states to a high-energy state from which it relaxes by emitting a single high-energy photon. This high-energy photon is returned to the host solar cell where it is absorbed. This approach increases the effective photon flux on the host cell, and thus the net current, while preserving a large open circuit voltage. A schematic single-junction solar cell equipped with an upconverter is shown in Figure 1(a) and a schematic upconversion process is shown in Figure 1(b). A key advantage of upconversion-backed PV systems is that the upconverter is electrically isolated from the host solar cell by an insulating layer acting as a transparent back contact. The electrical isolation of the upconversion layer avoids both the need for current matching between the host cell and upconversion layer and the degradation of the performance of the host cell due to the presence of mid-bandgap recombination centers.

Despite the conceptual simplicity, upconversion is difficult to implement because it always competes against both radiative and nonradiative loss processes. Upconversion has been observed in lanthanide and transition-metalion systems, [16, 17, 18, 19, quantum structures, 20, 21] and sensitized triplettriplet-annihilation (TTA) molecules. 22, 23, 22, Photovoltaic devices backed by upconversion materials have also been shown to achieve small peak efficiency 
a)

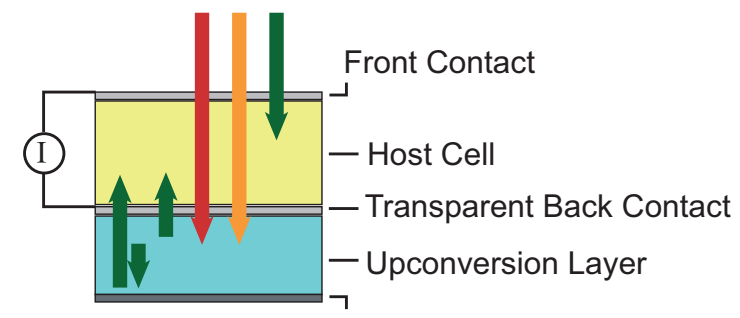

Reflective Layer

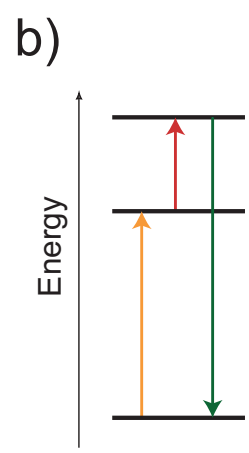

c)

d)
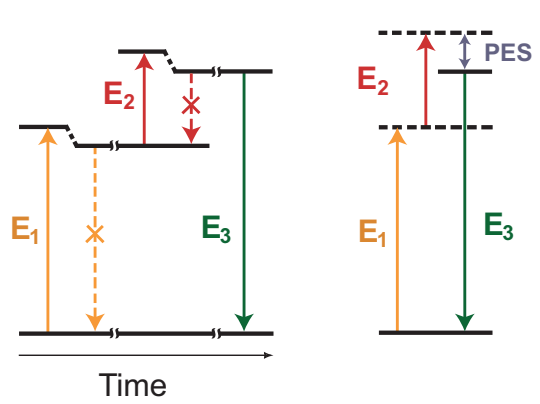

Figure 1: (Color Online) (a) Schematic diagram of a single-junction solar cell backed by an upconverter. High-energy photons (green) will be absorbed by the host solar cell. Low-energy photons (orange and red arrows) will pass through to the upconversion layer and be converted to high-energy photons that are then returned to the host solar cell. (b) Schematic depiction of perfect upconversion with no energy loss. (c) Schematic depiction of intentional energy loss used to suppress radiative and nonradiative loss. (d) Depiction of PES.

enhancements under solar concentration. 24] However, all existing photon upconversion materials (e.g. [19, 23]) have severe limitations: 1) only a narrow band of incident photon energies can be absorbed, 2) there is a low probability of upconversion and 3) much of the absorbed energy is lost as heat. We describe an approach that overcomes these limitations by using semiconductor materials with wide absorption bands and applying heterostructure engineering to make a small, controlled sacrifice of energy after each absorption step. This approach dramatically improves upconversion probability, minimizes the loss of energy to heat, and allows optical absorption and emission wavelengths to be tailored for PV or other applications. 


\section{Modeling Upconversion Materials and Systems}

The theoretical efficiency of a single junction solar cell backed by an upconverter has been modeled by Trupke[13] and Atre 14] using detailed balance methods. In their approach, the upconverter is modeled as a series of two small bandgap solar cells driving a light-emitting diode (LED). The two sub-cells have different bandgaps, enabling the upconverter to harvest two sub-bands of the solar spectrum in a manner analogous to both multi-junction solar cells and IBSCs. This model system allows the application of well-established detailed balance models for each of the upconverter sub-cells and standard diode equations for the LED. Using this approach, Trupke calculated a maximum theoretical device efficiency of $47.6 \%$ for nonconcentrated sunlight. 14 The results of Trupke and others demonstrate that significant gains in solar energy conversion efficiency could be obtained with upconversion-backed solar cells. However, detailed balance models consider only the bandgaps of hypothetical materials and do not provide metrics by which potential upconversion materials can be evaluated.

We define and consider three generalized performance metrics that capture the physical processes limiting the use of upconversion for PV device applications: absorption bandwidth (AB), upconversion quantum efficiency (UQE) and photon energy sacrifice (PES). AB is the spectral range of low-energy photons that can be absorbed by the upconverting material. UQE is the probability that a high-energy photon is produced from an absorbed pair of incident low-energy photons 1 The UQE is limited by all radiative and non-radiative recombination processes. In a simple three-level system, like the one depicted in Figure 1(b), the radiative loss pathways are impossible to suppress because the optical dipoles for absorption and emission of low-energy photons are equal. Suppress-

\footnotetext{
${ }^{1}$ Note that we define $100 \%$ UQE to be the highest possible quantum efficiency: 2 lowenergy photons are absorbed and 1 high-energy photon is emitted. Other reports in the literature define quantum efficiency in terms of photons out divided by photons in, resulting in a maximum quantum efficiency for upconversion of $50 \%$. We have adjusted all literature values to match our definition to allow fair comparisons.
} 
ing these radiative losses requires relaxation into a shelving state from which radiative emission is forbidden, as depicted in Figure 1(c). This relaxation process necessarily involves energy loss. We define PES as the minimum energy lost to heat during the upconversion process, analogous to the energy relaxation term defined by Atre. 14] As depicted in Figure 1(d), PES can also be understood as the difference between a) the sum of the lowest possible energies of the two incident photons and b) the energy of the emitted photon. Some PES is necessary for the upconversion process to occur, but large PES necessarily decreases the maximum overall solar energy conversion that can be achieved. As we will show, PES can be engineered to increase UQE and overall solar energy harvesting efficiency.

We employ a simple calculation to illustrate the impact of UQE and PES on solar energy conversion efficiency. A complete description of this calculation is provided in Sections IIA and B of [25]. Briefly, we calculate the host cell efficiency using well-established detailed balance methods, but increase the flux of photons incident on the host cell corresponding to the output of the upconversion material. The output of the upconversion material is simply computed by taking the number of low-energy photon pairs incident on the upconversion material in two wavelength bands (E1 and E2, see Figure 1(c) and (d)), assuming $100 \%$ absorption, and then multiplying by the UQE. The number of E1 and E2 photons incident on the upconverstion materials are calculated by integrating the solar spectrum over the range of wavelengths for each transition (e.g. from E2 to E1). As is typical for detailed balance models, we assume optimal spectral splitting and a continuous density of states above the band edge. For a given host cell bandgap $\left(\mathrm{E}_{g}\right)$ we vary $\mathrm{E} 1$ and $\mathrm{E} 2$ to find the maximum efficiency of the host cell subject to two constraints: E3>Eg and E3=E1+E2-PES.

This model, while simplistic, provides a clear framework by which we can evaluate the potential impact of UQE and PES on solar energy conversion efficiency. In Figure 2(a) we show the net solar energy conversion efficiency for host cells with bandgaps between $0.5 \mathrm{eV}$ and $4.5 \mathrm{eV}$ for a range of UQE values and a constant PES of $300 \mathrm{meV}$. Zero percent UQE corresponds to no upconversion 
and the results agree with the Shockley-Queisser limit of 33\% (black curve). With 100\% UQE (red curve), the peak efficiency reaches nearly $43 \%$, demonstrating the potential for significant improvements in solar energy conversion efficiency when UQE is high. Figure 2(b) shows net solar energy conversion efficiency as a function of host cell bandgap for a range of PES values between $150 \mathrm{meV}$ and $550 \mathrm{meV}$ assuming a constant UQE of $72.5 \%$. Even for the largest PES $(550 \mathrm{meV})$, the overall efficiency decreases by less than $5 \%$ compared to the smallest PES value evaluated. Taken together, Figure 2(a) and (b) demonstrate that a significant increase in net solar energy conversion efficiency could be realized for appropriate values of UQE and PES and that the overall efficiency is more sensitive to UQE than to PES. This result tells us that increases in PES that enable increased UQE are advantageous. We note that one crucial aspect missing from this model is the correlation between UQE and PES that originates in the photophysics of photon absorption and carrier relaxation. Because the details of these processes are material specific, our detailed balance model cannot, by itself, be used to predictively model upconversion materials. We return to this point below.

\section{Analysis of existing upconversion materials}

The generalized metrics AB, UQE and PES provide a framework for evaluating the potential solar energy impact of candidate upconversion materials. The most common photon upconversion material systems are those based on lanthanide or rare earth metals (highest UQE reported 2 4-20\% 27, 28, 29, 17, [30]), and those based on sensitized triplet-triplet annihilation (TTA) of organic molecules (highest UQE reported: 26-35\% 31, 32, 33, 34, 35]). The high UQE values reported for TTA upconverters are due in large part to the rapid transfer of charge to a long-lived triplet state via intersystem crossing, which reduces

\footnotetext{
${ }^{2}$ Lanthanide-based UQE values from literature have been adjusted by multiplying by a factor of 2 to be consistent with our definition of UQE.
} 

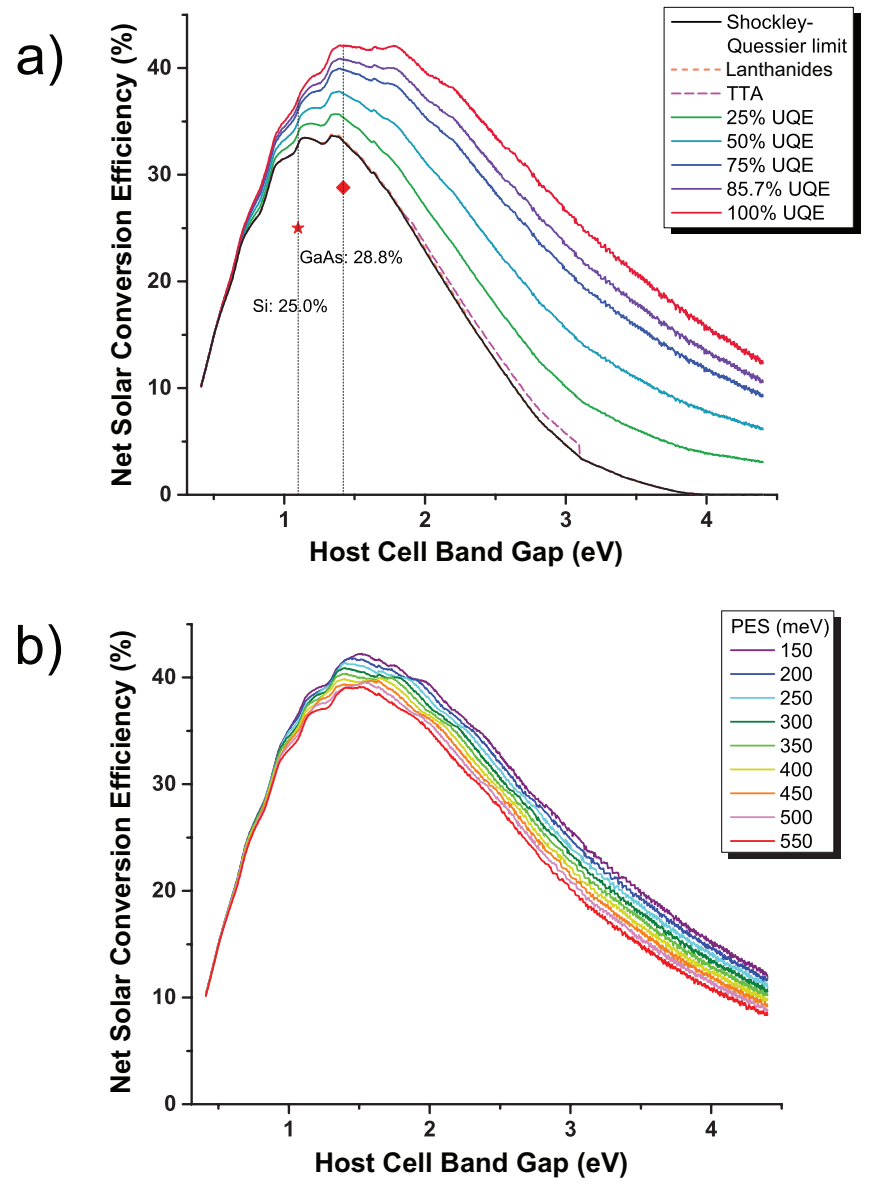

Figure 2: (Color Online)Upconversion-backed PV device efficiencies as a function of host cell bandgap. Top: Different UQE with fixed PES $(300 \mathrm{meV})$. The star and diamond represent the highest reported efficiencies for single-junction Si and GaAs PV cells [26]; Bottom: Different PES with fixed UQE (72.5\%)

radiative and nonradiative recombination. This long-lived triplet state is analogous to the shelving states depicted in Fig. 1.(c). Although Figure 2 suggests that a UQE of $\leq 35 \%$ would permit these upconversion materials to marginally improve net solar energy conversion efficiency, their utilization of the solar spectrum and/or high PES values make their potential PV impact much smaller. We have summarized the potential impact of these upconversion materials in 
Table 1 and now provide a more detailed analysis of their limitations.

First, the atomic transitions of the sensitizer molecule only allow absorption of photons within a narrow spectral window (small AB). Moreover, both lowenergy photons must be absorbed within the same spectral window. As a result, it is not possible for molecular upconversion materials to harvest three separate portions of the solar spectrum in the manner assumed by detailed balance models of upconversion-based PV devices. Second, most existing lanthanide and TTA upconverters emit photons in energy ranges that are incompatible with existing PV technologies (i.e. potential host PV cells) and similarly absorb photons in energy ranges above what would be useful for upconversion-backed PV. Those that absorb and emit in solar-relevant wavelength ranges are limited by their absorption of a narrow spectral band and low quantum efficiency[36]. To illustrate this point, we have used our detailed balance model (described above) to calculate the projected maximum solar energy conversion efficiency for a host single-junction PV cell backed by a TTA or lanthanide upconverter as a function of the host cell bandgap (purple and orange dashed lines in Figure2(a)). We use $\mathrm{AB}$ and $\mathrm{PES}$ values based on typical absorption and emission energies reported for TTA and lanthanide upconverters. We also use, for each system, the highest UQE values reported in literature to date. These parameters and their references are given in Table 1. For lanthanide-backed devices our model predicts a negligible increase in efficiency irrespective of host cell bandgap, shown by the orange dashed line in Figure 2(a). As an example, for a GaAs host cell $\left(\mathrm{E}_{g}=\right.$ $1.42 \mathrm{eV}$ ) our model predicts an improvement from 33\% (SQ limit) to $33.4 \%$ when backed by a $\mathrm{NaYF}_{4}: \mathrm{Er}^{3+} / \mathrm{Yb}^{3+}$ upconverter.

For TTA backed devices we predict a slightly larger increase in efficiency shown by the purple dashed line in Figure 2(a). One of the highest reported UQE values for TTA molecules used a DPA (9,10-diphenylanthracene) emitter (emitting at $2.85 \mathrm{eV}$ ), combined with palladium(II) tetraphenylporphyrin as a triplet sensitizer (absorbing at $2.37 \mathrm{eV}$ ). Such an emitter/sensitizer pair would be compatible with a $2.75 \mathrm{eV}$ host cell. According to the SQ limit, a singlejunction PV cell with a bandgap of $2.75 \mathrm{eV}$ can have a maximum efficiency of 
only $8.01 \%$. Our model allows us to estimate that addition of this upconversion material to a $2.75 \mathrm{eV}$ host cell would increase the net solar energy conversion from $8.01 \%$ to $8.99 \%$. Our model further allows us to estimate that the net solar energy conversion efficiency of a $2.75 \mathrm{eV}$ host cell backed by a hypothetical TTA upconversion material with $100 \%$ efficiency would be only $10.8 \%$. Although this is a significant increase in device efficiency, the net efficiency remains below what can already be achieved with conventional single junction materials. In other words, the spectral utilization of existing TTA materials makes them impractical for high efficiency photovoltaics even if their UQE could be improved substantially.

This example highlights the intrinsic limitations of molecular upconverters. Absorption of low-energy photons and emission of high-energy photons are governed by the atomic energy levels of the molecule. The narrow $\mathrm{AB}$ of both lanthanide and TTA-based materials for photon upconversion means that only a small fraction of the incident solar photons with energy below the host cell bandgap can be absorbed. Moreover, the low UQE and large PES mean that much of the absorbed energy is lost as either heat or re-radiated low-energy photons that cannot be harvested by the host PV cell. As a result, the maximum theoretical solar energy conversion efficiency that could be achieved using the best molecular upconverter materials available is $<20 \%$, drastically worse than both the $46.7 \%$ predicted by detailed balance calculations 13 and existing single junction PV cells. In the language of our generalized framework, molecular upconverters have $\mathrm{AB}$ and $\mathrm{UQE}$ values that are too small to make a meaningful impact on PV device technologies. Moreover, even the best UQE values $(\sim 35 \%)$ that have been achieved come at the expense of extremely large PES values.

\section{A New Paradigm for Upconversion Materials}

Guided by the analysis associated with Figure 2, we now develop a design for a new upconversion nanostructure. This new nanostructure design utilizes 


\begin{tabular}{|c|c|c|}
\hline & Triplet-triplet Annihilation (TTA) & Lanthanide \\
\hline Sensitizer/Emitter & $\begin{array}{c}\mathrm{Pt}(\mathrm{II}) \text { or } \mathrm{Pd}(\mathrm{II}) \text { porphyrin } / 9,10- \\
\text { diphenylanthracene (DPA) }\end{array}$ & $\begin{array}{c}\mathrm{NaYF}_{4} \text { co-doped with } \mathrm{Er}^{3+} \text { and } \\
\mathrm{Yb}^{3+}\end{array}$ \\
\hline $\mathrm{AB}$ & $0.09 \mathrm{eV} 37$ & $0.13 \mathrm{eV} 38$ \\
\hline UQE & \begin{tabular}{llllll|}
$28-36 \%$ & 31 & 32 & 33 & 34 \\
\end{tabular} & $4-12 \% *$ [27 $28,29,17$ \\
\hline $\begin{array}{c}\text { PES } \\
\operatorname{Min}(\operatorname{Max})\end{array}$ & $0.54(1.34) \mathrm{eV}$ & $0.16(0.47) \mathrm{eV}$ \\
\hline $\begin{array}{l}\text { Device Efficiency } \\
\text { Projected }\end{array}$ & $\begin{array}{c}\text { see Figure } 2 \text { a) } \\
\text { (purple dashed line) }\end{array}$ & $\begin{array}{c}\text { see Figure } 2 \text { a) } \\
\text { (orange dashed line) }\end{array}$ \\
\hline $\begin{array}{l}{ }^{*} \mathrm{BaY}_{4} \mathrm{~F}_{8}: \mathrm{Er}^{3+} \text { has } \\
\text { ficiencies for } \mathrm{Si} \text { cells } \\
\mathrm{NaYF}_{4}: \mathrm{Er}^{3+} / \mathrm{Yb}^{3+} \text {. } \\
4 \% \text { to } 12 \% \text {. }\end{array}$ & $\begin{array}{l}\text { en reported to have a UQE of } \mathrm{u} \\
\text { acked by } \mathrm{BaY}_{4} \mathrm{~F}_{8}: \mathrm{Er}^{3+} \text { remain } \mathrm{b} \\
\text { Te therefore report } \mathrm{UQE} \text { values for }\end{array}$ & $\begin{array}{l}\text { o } 20 \% \text { 30. However, calculate } \\
\text { w those of the GaAs cell backe } \\
\mathrm{aYF}_{4}: \mathrm{Er}^{3+} / \mathrm{Yb}^{3+} \text {, which range }\end{array}$ \\
\hline
\end{tabular}

Table 1: Existing upconverters and corresponding absorption bandwidth (AB), upconversion quantum efficiency (UQE), and photon energy sacrifice (PES), as well as calculated device efficiency. The reported UQE values for lanthanides were expressed as the number of photons emitted divided by the number of photons absorbed. For use in the projected device efficiency calculation these values were multiplied by a factor of 2 to account for the two-photon process, as described in the text.

controlled band offsets and gradients to take advantage of solid state absorption properties and enable high UQE with a low PES and large AB. We note that the band structure we propose can be realized in many material systems, including colloidally-synthesized nanoparticles. For clarity, we present one specific implementation of the design in a III-V material platform. A schematic band diagram for our new upconversion nanostructure is shown in Figure 3 . The active area of the device is bounded by InAlGaAs to confine all optically-generated carriers. Both low-energy photons are absorbed within an InAs quantum dot (QD). The first absorbed low-energy photon promotes an electron from the valence band to the lowest-energy confined state of the QD (process 1). Radiative recombination of the optically-generated electron-hole pair is suppressed by the rapid escape of the hole (process 2), which is facilitated by the zero valence band offset and the built-in quasi-electric field [39] due to the compositional gradient in the InAlBiAs layer that caps the QD. The valence band gradient that drives hole escape from the QD implements the first intentional 'PES in the valence band' (PVB), 
which sacrifices energy to prevent thermal excitation of the hole back to the QD where radiative recombination with the trapped electron would be possible. The second absorbed low-energy photon promotes the confined electron from the lowest energy state of the QD to a bulk conduction band state (process 3). Radiative and non-radiative relaxation of this excited electron can be largely suppressed so long as the rate of escape from the QD (process 4) is fast relative to the fastest loss pathway. The discrete density of states in the QD creates a bottleneck for phonon relaxation to the QD ground state, with typical relaxation times of 10s of picoseconds. [40] Thermal relaxation through the continuum of states of the bulk conduction band is typically of order 1 picosecond, making electron escape the dominant process. 41, 42, The second intentional PES is the conduction band gradient (PCB) of the InAlBiAs layer, which guides the electron to the recombination zone where it can recombine with the hole to emit a high-energy photon (process 5). Again, a PCB energy sacrifice, implemented by the conduction band gradient, prevents thermal escape of the electrons from the recombination zone. We note that all of these radiative recombination and carrier escape processes are quantitatively included in the computational model we use to estimate the performance of this device, as described below and in [25].

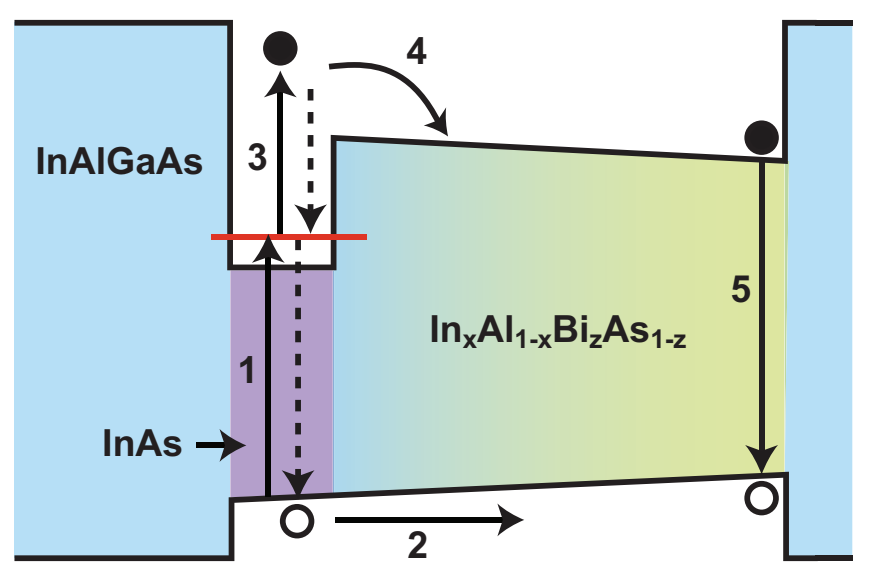

Figure 3: (Color Online) Upconversion nanostructure electronic structure 
The proposed upconversion nanostructure is an all-optical device that funnels both electrons and holes to the same recombination zone and does not involve any net current flow. In this sense, the upconverting nanostructure functions in a manner fundamentally different from solid state PV devices that require generation of quasi-Fermi levels to drive current flow through an external circuit. We note that both low-energy photon absorption processes involve a transition between a continuum of states and a discrete state of the QD (e.g. process 1: valence band to discrete QD state, process 3: discrete QD state to conduction band). Consequently, both low-energy photon transitions are able to absorb a continuum of solar photons with energy above the transition threshold. The $\mathrm{AB}$ for this device is thus expected to be approximately $0.8 \mathrm{eV}$, as described in [25].

\section{Estimating performance}

To understand system-level trade-offs that guide the design criteria for useful upconversion nanoparticles, and to identify precise design criteria for the specific nanoparticle realization discussed here, we have developed a computational model for both this upconversion nanostructure and a PV system backed by this upconverter. This analysis takes into account the impact of non-ideal $\mathrm{AB}, \mathrm{UQE}$ and PES values. In the first step we employ a rate equation model of our upconversion nanostructure design to compute the equilibrium output of high-energy photons as a function of PES under one-sun illumination. The internal $\mathrm{UQE}$ is then computed from the ratio of emitted high-energy photons to absorbed low-energy photon pairs. This model, described in detail in [25], uses numerical values for parameters such as the absorption cross section of specific

optical transitions and the rates of all carrier generation, relaxation, transport, and recombination processes. The values we use for such parameters are taken from literature for material platforms similar to those discussed later in this paper. We assume, for purposes of the model, that these parameter values are independent of the energy of the optical transitions or any changes in the mate- 
rial platform that might be necessary to realize upconversion over a wide range of host cell bandgaps. While this assumption certainly limits the quantitative accuracy of the model, it provides a framework by which we can numerically evaluate the impact of the dependence of UQE on PES in terms of net solar energy conversion efficiency.

The inset to Figure 4(a) plots the computed internal UQE of the upconversion nanostructure described here as a function of PES. This result confirms that PES and internal UQE are not independent parameters - higher PES enables carriers to more efficiently funnel to the recombination zone, resulting in higher UQE. We see that a UQE as high as $96 \%$ should be achievable with a PES of $550 \mathrm{meV}$. We note that this maximum UQE value is a consequence of the QD density of states in this specific realization of the new upconversion paradigm and should not be viewed as a fundamental limit. The main panel of Figure 4 (a) plots UQE as a function of both PES and host cell bandgap, which constrains the bandgaps E1 and E2. The results show that a UQE of approximately $96 \%$ can be obtained for PES values of order $500 \mathrm{meV}$ for all host cell bandgaps. However, maximum UQE does not necessarily correspond to maximum solar energy conversion efficiency for an upconversion-backed PV system.

To estimate the net solar energy conversion efficiency that could be achieved with this new upconversion paradigm, we must make additional assumptions about the PV harvesting device that incorporates the upconverter. We assume that the upconversion layer (see Figure 1) is sufficiently thick to absorb all incident low-energy photons in the appropriate ranges of the solar spectrum. For example, 10 or more layers of QDs are necessary for adequate photocurrent generation in intermediate band solar cell devices, which utilize the solar spectrum in a similar fashion. 43. We further assume that the external UQE of the upconversion layer is equal to the internal $\mathrm{UQE}$ we compute for a single upconversion nanostructure. Future models will certainly need to consider device-level performance losses that arise from photon propagation through interfaces and within the upconversion material. Here we use a simpler model to consider the optimal tradeoff between UQE and PES for maximum solar energy conversion with this 
upconversion nanostructure design.

Figure 4 (b) plots the net solar energy conversion efficiency of a host PV cell backed by our upconversion material as a function of both the bandgap of the host cell and the PES value. The computation of net solar energy conversion efficiency includes the UQE associated with each combination of PES and host cell bandgap as obtained from the rate equation model whose results are shown

in Figure 4(a). Figure 4(b) shows that net solar energy conversion efficiencies exceeding 39\% could be realized with PES values ranging from 390 to $550 \mathrm{meV}$ by using host PV cells with bandgaps between 1.3 and $1.75 \mathrm{eV}$. This range of relatively constant net solar energy conversion efficiency is a result of the tradeoff between UQE and PES: higher UQE increases the number of highenergy photons emitted for a given number of incident low-energy photons, but increasing PES reduces the number of low-energy solar photons that can be harvested by the upconversion material. We note that GaAs, with a bandgap of $1.42 \mathrm{eV}$, lies within the window of host cell bandgaps for which overall high efficiency improvements can be achieved.

We stress that the calculation of net solar energy harvesting efficiency and optimal host cell bandgaps depends critically on the relationship between UQE and PES and is thus specific to upconversion materials whose performance can be described by the rate equation model used here. System-integration challenges such as guiding high-energy photons to the energy harvesting system and optimizing the host cell for performance under higher fluxes of high-energy photons are not considered here.

\section{Feasibility}

To further demonstrate the feasibility of this upconversion approach, we show that this nanostructure can be fabricated by incorporating a small amount of bismuth into conventional III-V semiconductors (GaAs, InGaAs, InAlAs). This category of materials, known as dilute bismuthides or dilute bismides, includes GaBiAs, InGaBiAs and related materials that have been grown by 

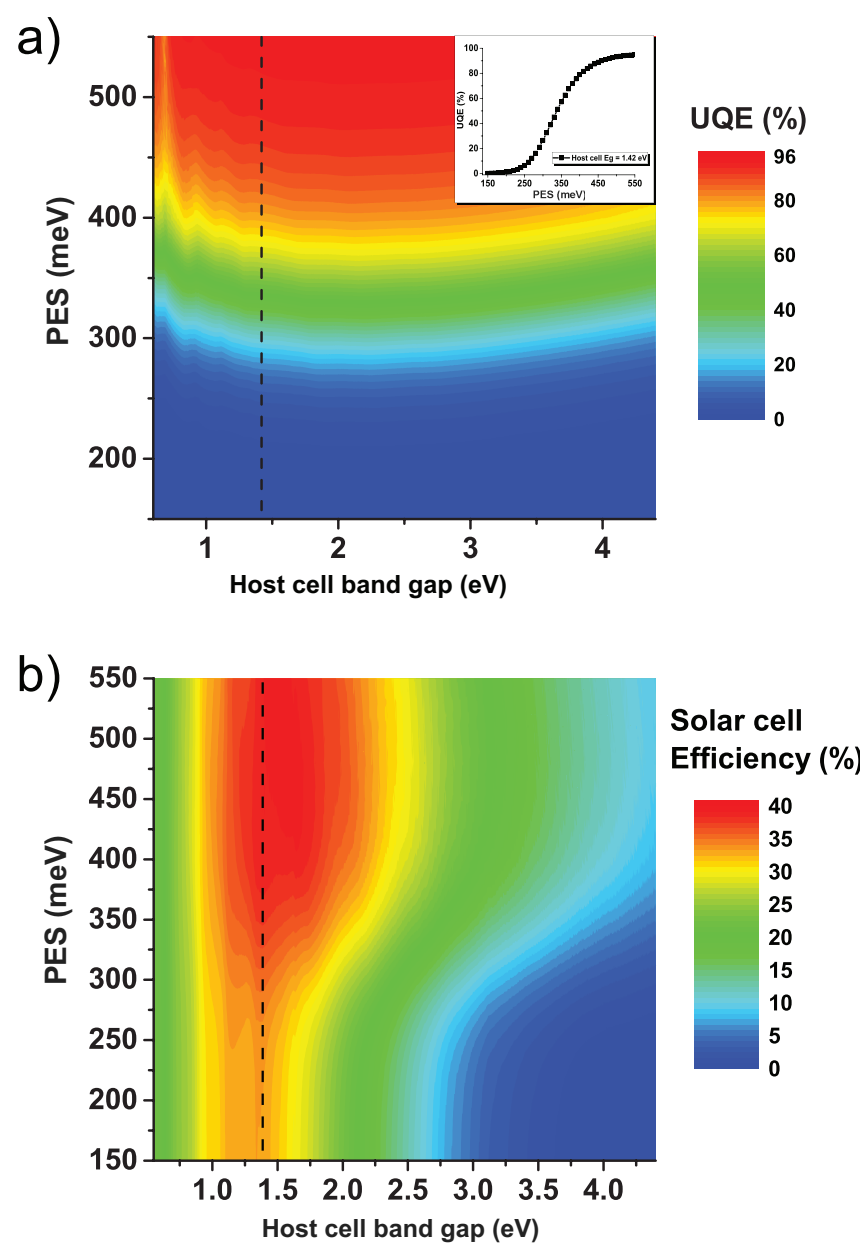

Figure 4: (Color Online) Contour plot of a) internal UQE computed for a single upconversion nanostructure and b) Efficiency, as a function of PES and host cell bandgap. Inset to (a) shows UQE vs PES from the rate equation model. Dotted black line indicates host cell bandgap of $1.42 \mathrm{eV}$, showing high achievable net solar cell efficiencies with GaAs host cells

MBE, 44, 45, 46] MOVPE, 47] and LPE. 48] Incorporation of bismuth in III-V materials is known to cause valence band anticrossing (VBAC) that raises the valence band [41, 42, 46] without significantly affecting the conduction band of the matrix material. Adjusting the ratio between the group III elements controls both the conduction band and valence band edges. Simultaneously controlling both the group III ratio and the bismuth concentration therefore provides inde- 
pendent tuning of the conduction and valence bands. This tunability allows the simultaneous creation of a zero valence band offset with the QD and a gradient in conduction and valence bands that drives carriers to the recombination zone.

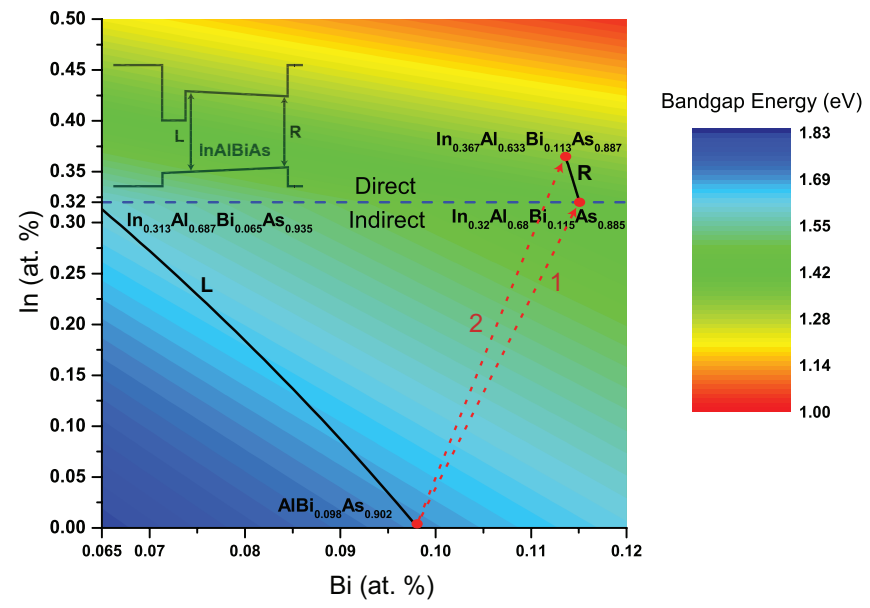

Figure 5: (Color Online) Simulation of the bandgap energy of InAlBiAs as a function of composition. Compositions for the left (L) barrier indicated by the black line satisfy the zero valence band offset between the InAs VB and the barrier VB energy. Bandgap energies range between 1.61 and $1.70 \mathrm{eV}$. Barrier compositions for the right $(\mathrm{R})$ line indicated by the corresponding black line satisfy a fixed 185meV PVB. Eg ranges between 1.33 and $1.43 \mathrm{eV}$. The composition gradient from the left to the right side of the InAlBiAs zone is indicated by red dotted lines 1 and 2, corresponding to an upconverter backing a $1.42 \mathrm{eV}$ and $1.325 \mathrm{eV}$ host cell, respectively. The inset schematic is intended to aid the reader in understanding which compositional profiles will be used at specific points in the upconversion nanostructure. Note that regions with direct vs. indirect bandgap are indicated.

To show that the desired band alignments and bandgaps can be achieved in the InAlBiAs system, we compute two possible compositional profiles that can implement this device design. We calculate the desired compositions for both sides of the InAlBiAs region using a simple VBAC model based on perturbation theory [47. A detailed description of the material parameters and design criteria can be found in [25]. The results of our bandgap calculations are shown in Figure 5. The lower black line in Figure 5 (marked L) indicates the range of InAlBiAs compositions that satisfy the constraints of zero valence band offset 
with the QD. All compositions along this line have an indirect bandgap, which minimizes the possibility of radiative recombination in this region. The upper black line in Figure 5 (marked R) shows the range of possible compositions for a recombination zone with a $185 \mathrm{meV}$ valence band offset (PVB) relative to the InAlBiAs alloy at the interface with the QD. These compositions all have a direct bandgap, enhancing radiative recombination of electrons and holes.

To demonstrate compatibility with existing PV host cell materials, we first assume the upconverter backs a GaAs host cell (1.42 eV bandgap) and has a $280 \mathrm{meV}$ PES (185 meV PVB and $95 \mathrm{meV} \mathrm{PCB}$ ). For this implementation, the composition parameters are chosen so that the upconversion nanostructure emits photons at $1.426 \mathrm{eV}$, just above the GaAs bandgap of $1.42 \mathrm{eV}$. The desired composition gradient for the InAlBiAs zone in this case is indicated by the dashed red line labeled 1 . The resulting net solar conversion efficiency is $34.6 \%$, exceeding the SQ limit. To show that the InAlBiAs system can enable efficiencies approaching the $39 \%$ limit we predict in Section 5, we report composition parameters for the optimal net solar cell efficiency case, which is when the upconverter backs a $1.325 \mathrm{eV}$ bandgap host cell. In this case the bismuthide layer implements a $370 \mathrm{meV}$ PES (185meV PVB and $185 \mathrm{meV}$ PCB) and emits photons with energies of $1.33 \mathrm{eV}$. The desired composition gradient for this case is indicated by the dashed red line labeled 2 and results in a net solar energy conversion efficiency of $38.4 \%$ We note that this photon upconversion paradigm could be physically realized with many material combinations and is not limited to the InAlBiAs system introduced here as a demonstration of feasibility.

\section{Conclusion}

We have summarized the important features of upconverting materials with relevance to solar energy harvesting. We have further shown that existing upconversion materials have drastically different performance metrics than the upconverters used in existing detailed balance calculations of the solar energy conversion efficiency that can be achieved with upconversion-backed PV cells. 
We propose a new design for a nanostructured solid state upconverter optimized for solar energy harvesting. The design uses heterostructure engineering to maximize net solar energy conversion efficiency while minimizing PES and maximizing $\mathrm{AB}$. We demonstrate the feasibility of this design by calculating specific compositions of InAlBiAs alloys that realize the required bandgaps and alignments. Experimental measurement and optimization of UQE, PES and $\mathrm{AB}$ is a critical next step in the development of the proposed upconversion nanostructures and efforts are underway. High-performance photon upconversion materials will enable new functionality and improved performance not only in photovoltaics but also for technologies that include: photoelectrochemical and photocatalytic systems, energy efficient displays and lighting, medical imaging, optogenetic manipulation, and disease treatment. The above work lays the foundation for systematic design of efficient upconversion materials for all of these applications.

\section{Acknowledgement}

The authors acknowledge financial support from the University of Delaware Energy Institute, the Energy Efficiency and Renewable Energy program of the Department of Energy and the W.M. Keck Foundation.

[1] W. Shockley, H. Queisser, Detailed balance limit of efficiency of p-n junction solar cells, Journal of Applied Physics 32 (3) (1961) 510-519.

[2] R. D. Schaller, V. I. Klimov, High efficiency carrier multiplication in PbSe nanocrystals: implications for solar energy conversion, Physical review letters 92 (18) (2004) 186601.

[3] O. E. Semonin, J. M. Luther, S. Choi, H.-Y. Chen, J. Gao, A. J. Nozik, M. C. Beard, Peak external photocurrent quantum efficiency exceeding $100 \%$ via MEG in a quantum dot solar cell, Science 334 (6062) (2011) 1530-1533. 
[4] J. Geisz, D. Friedman, J. Ward, A. Duda, W. Olavarria, T. Moriarty, J. Kiehl, M. Romero, A. Norman, K. Jones, 40.8\% efficient inverted triplejunction solar cell with two independently metamorphic junctions, Applied Physics Letters 93 (12) (2008) 123505.

[5] W. Guter, J. Schone, S. P. Philipps, M. Steiner, G. Siefer, A. Wekkeli, E. Welser, E. Oliva, A. W. Bett, F. Dimroth, Current-matched triplejunction solar cell reaching $41.1 \%$ conversion efficiency under concentrated sunlight, Applied Physics Letters 94 (22) (2009) 223504-223503.

[6] V. Sabnis, H. Yuen, M. Wiemer, High-efficiency multijunction solar cells employing dilute nitrides, AIP Conference Proceedings 1477 (2012) 14.

[7] A. Luque, A. Martí, C. Stanley, Understanding intermediate-band solar cells, Nature Photonics 6 (3) (2012) 146-152.

[8] A. Luque, A. Martí, The intermediate band solar cell: progress toward the realization of an attractive concept., Advanced Materials 22 (2) (2010) 160-74. doi:10.1002/adma.200902388.

URL http://www.ncbi.nlm.nih.gov/pubmed/20217682

[9] A. Luque, A. Mart, C. Stanley, N. Lopez, L. Cuadra, D. Zhou, J. L. Pearson, a. McKee, General equivalent circuit for intermediate band devices: Potentials, currents and electroluminescence, Journal of Applied Physics 96 (1) (2004) 903. doi:10.1063/1.1760836.

URL http://scitation.aip.org/content/aip/journal/jap/96/1/10. 1063/1.1760836

[10] N. Lopez, L. Reichertz, K. Yu, K. Campman, W. Walukiewicz, Engineering the electronic band structure for multiband solar cells, Physical Review Letters 106 (2011) 028701.

[11] M. J. Keevers, M. A. Green, Efficiency improvements of silicon solar cells by the impurity photovoltaic effect, Journal of Applied Physics 75 (8) (1994) 4022. doi:10.1063/1.356025. 
URL http://scitation.aip.org/content/aip/journal/jap/75/8/10. $1063 / 1.356025$

[12] D. G. Sellers, S. Polly, S. M. Hubbard, M. F. Doty, Analyzing carrier escape mechanisms in InAs/GaAs quantum dot p-i-n junction photovoltaic cells, Applied Physics Letters 104 (2014) 223903.

[13] T. Trupke, M. A. Green, P. Wurfel, Improving solar cell efficiencies by upconversion of sub-bandgap light, Journal of Applied Physics 92 (7) (2002) $4117-4122$.

[14] A. C. Atre, J. Dionne, Realistic upconverter-enhanced solar cells with nonideal absorption and recombination efficiencies, Journal of Applied Physics 110 (3) (2011) 034505-034505.

[15] J. A. Briggs, A. C. Atre, J. A. Dionne, Narrow-bandwidth solar upconversion: Case studies of existing systems and generalized fundamental limits, Journal of Applied Physics 113 (12) (2013) 124509-124509.

[16] J.-C. Boyer, L. A. Cuccia, J. A. Capobianco, Synthesis of colloidal upconverting $\mathrm{NaYF}_{4}: \mathrm{Er}^{3+} / \mathrm{Yb}^{3+}$ and $\mathrm{Tm}^{3+} / \mathrm{Yb}^{3+}$ monodisperse nanocrystals, Nano Letters 7 (3) (2007) 847-852.

[17] S. Fischer, J. Goldschmidt, P. Loper, G. Bauer, R. Bruggemann, K. Kramer, D. Biner, M. Hermle, S. Glunz, Enhancement of silicon solar cell efficiency by upconversion: Optical and electrical characterization, Journal of Applied Physics 108 (4) (2010) 044912-044912.

[18] S. Heer, K. Kömpe, H. Güdel, M. Haase, Highly efficient multicolour upconversion emission in transparent colloids of lanthanide-doped $\mathrm{NaYF}_{4}$ nanocrystals, Advanced Materials 16 (2324) (2004) 2102-2105.

[19] Z. Li, Y. Zhang, S. Jiang, Multicolor core/shell-structured upconversion fluorescent nanoparticles, Advanced Materials 20 (2008) 4765-4769. 
[20] D. Tex, I. Kamiya, Upconversion of infrared photons to visible luminescence using InAs-based quantum structures, Physical Review B 83 (8) (2011) 081309 .

[21] X. Wang, W. W. Yu, J. Zhang, J. Aldana, X. Peng, M. Xiao, Photoluminescence upconversion in colloidal CdTe quantum dots, Physical Review B 68 (12) (2003) 125318.

[22] T. N. Singh-Rachford, A. Nayak, M. L. Muro-Small, S. Goeb, M. J. Therien, F. N. Castellano, Supermolecular-chromophore-sensitized nearinfrared-to-visible photon upconversion, Journal of the American Chemical Society 132 (40) (2010) 14203-14211.

[23] Y. Y. Cheng, B. Fückel, T. Khoury, R. G. Clady, M. J. Tayebjee, N. EkinsDaukes, M. J. Crossley, T. W. Schmidt, Kinetic analysis of photochemical upconversion by triplet-triplet annihilation: Beyond any spin statistical limit, The Journal of Physical Chemistry Letters 1 (12) (2010) 1795-1799.

[24] Y. Y. Cheng, B. Fückel, R. W. MacQueen, T. Khoury, R. G. Clady, T. F. Schulze, N. Ekins-Daukes, M. J. Crossley, B. Stannowski, K. Lips, Improving the light-harvesting of amorphous silicon solar cells with photochemical upconversion, Energy \& Environmental Science 5 (5) (2012) 6953-6959.

[25] E. Y. Chen, J. Zhang, D. G. Sellers, Y. Zhong, J. M. O. Zide, M. F. Doty, A kinetic rate model of novel upconversion nanostructures for high-efficiency photovoltaics, Photovoltaics, IEEE Journal of. Accepted.

[26] M. A. Green, K. Emery, Y. Hishikawa, W. Warta, E. D. Dunlop, Solar cell efficiency tables (Version 45), Progress in Photovoltaics: Research and Applications 23 (1) (2015) 1-9. doi:10.1002/pip. 2573.

URL http://dx.doi.org/10.1002/pip.2573

[27] A. Bril, J. L. Sommerdijk, A. W. de, Jager, On the efficiency of $\mathrm{Yb}^{3+}$ $\mathrm{Er}^{3+}$ activated up-conversion phosphors, Journal of The Electrochemical Society 122 (5) (1975) 660-663. 
[28] R. H. Page, K. I. Schaffers, P. A. Waide, J. B. Tassano, S. A. Payne, W. F. Krupke, W. K. Bischel, Upconversion-pumped luminescence efficiency of rare-earth-doped hosts sensitized with trivalent ytterbium, Journal of the Optical Society of America B 15 (3) (1998) 996-1008.

[29] J.-C. Boyer, F. C. J. M. van Veggel, Absolute quantum yield measurements of colloidal $\mathrm{NaYF}_{4}$ : $\mathrm{Er}^{3+}, \mathrm{Yb}^{3+}$ upconverting nanoparticles, Nanoscale 2 (8) (2010) 1417-1419.

[30] S. Fischer, E. Favilla, M. Tonelli, J. C. Goldschmidt, Record efficient upconverter solar cell devices with optimized bifacial silicon solar cells and monocrystalline BaY2F8:30\% Er3+ upconverter, Solar Energy Materials and Solar Cells 136 (2015) 127-134. doi:http://dx.doi.org/10.1016/j.solmat.2014.12.023. URL http://www.sciencedirect.com/science/article/pii/ S0927024814006709

[31] R. S. Khnayzer, J. Blumhoff, J. A. Harrington, A. Haefele, F. Deng, F. N. Castellano, Upconversion-powered photoelectrochemistry., Chemical communications (Cambridge, England) 48 (2) (2012) 209-11. doi: $10.1039 / \mathrm{c} 1 \mathrm{cc} 16015 \mathrm{j}$

URL http://www.ncbi.nlm.nih.gov/pubmed/22080384

[32] X. Cao, B. Hu, P. Zhang, High upconversion efficiency from hetero triplettriplet annihilation in multiacceptor systems, The Journal of Physical Chemistry Letters 4 (14) (2013) 2334-2338.

[33] B. Wang, B. Sun, X. Wang, C. Ye, P. Ding, Z. Liang, Z. Chen, X. Tao, L. Wu, Efficient triplet sensitizers of palladium (II) tetraphenylporphyrins for upconversion-powered photoelectrochemistry, The Journal of Physical Chemistry C 118 (3) (2014) 1417-1425.

[34] P. Duan, N. Yanai, N. Kimizuka, Photon upconverting liquids: matrix-free molecular upconversion systems functioning in air, Journal of the American Chemical Society 135 (51) (2013) 19056-19059. 
[35] J.-H. Kim, F. Deng, F. N. Castellano, J.-H. Kim, High efficiency low-power upconverting soft materials, Chemistry of Materials 24 (12) (2012) 22502252.

[36] B. Richards, A. Shalav, Enhancing the near-infrared spectral response of silicon optoelectronic devices via up-conversion, Electron Devices, IEEE Transactions on 54 (10) (2007) 2679-2684. doi:10.1109/TED.2007. 903197.

[37] R. Vadrucci, C. Weder, Y. C. Simon, Low-power photon upconversion in organic glasses, Journal of Materials Chemistry C 2 (16) (2014) 2837-2841.

[38] C. Renero-Lecuna, R. Martin-Rodriguez, R. Valiente, J. Gonzalez, F. Rodriguez, K. W. Kramer, H. U. Gudel, Origin of the high upconversion green luminescence efficiency in $\beta-\mathrm{NaYF}_{4}: 2 \% \mathrm{Er}^{3+}, 20 \% \mathrm{Yb}^{3+}$, Chemistry of Materials 23 (15) (2011) 3442-3448.

[39] H. Kroemer, Nobel Lecture: Quasielectric fields and band offsets: teaching electrons new tricks, Rev. Mod. Phys. 73 (3) (2001) 783-793. doi:10. 1103/RevModPhys.73.783.

URL http://link.aps .org/doi/10.1103/RevModPhys.73.783

[40] R. Heitz, H. Born, F. Guffarth, O. Stier, A. Schliwa, A. Hoffmann, D. Bimberg, Existence of a phonon bottleneck for excitons in quantum dots, Physical Review B 64 (24) (2001) 241305.

[41] D. B. Malins, a. Gomez-Iglesias, S. J. White, W. Sibbett, a. Miller, E. U. Rafailov, Ultrafast electroabsorption dynamics in an InAs quantum dot saturable absorber at $1.3 \mu \mathrm{m}$, Applied Physics Letters 89 (17) (2006) 171111. doi:10.1063/1.2369818.

URL http://scitation.aip.org/content/aip/journal/apl/89/17/ $10.1063 / 1.2369818$

[42] T. N. Leite, H. P. de Oliveira, Tunneling Processes in a Triangular Multibarrier Semiconductor Heterostructure, IEEE Transactions on Electron 
Devices 58 (3) (2011) 716-719. doi:10.1109/TED.2010.2100399.

URL http://ieeexplore.ieee.org/lpdocs/epic03/wrapper.htm? arnumber $=5682401$

[43] A. Luque, A. Marti, The intermediate band solar cell: Progress toward the realization of an attractive concept, Advanced Materials 22 (2010) 160-174.

[44] X. Lu, D. A. Beaton, R. B. Lewis, T. Tiedje, Y. Zhang, Composition dependence of photoluminescence of $\mathrm{GaAs}_{1 x} \mathrm{Bi}_{x}$ alloys, Applied Physics Letters 95 (4) (2009) 041903. doi:10.1063/1.3191675.

URL http://scitation.aip.org/content/aip/journal/apl/95/4/10. 1063/1.3191675

[45] Y. Zhong, P. B. Dongmo, J. P. Petropoulos, J. M. O. Zide, Effects of molecular beam epitaxy growth conditions on composition and optical properties of $\operatorname{In}_{x} \mathrm{Ga}_{1-x} \mathrm{Bi}_{y} \mathrm{As}_{1-y}$, Applied Physics Letters 100 (11) (2012) 112110112114 .

[46] J. P. Petropoulos, Y. Zhong, J. M. O. Zide, Optical and electrical characterization of ingabias for use as a new mid-infrared optoelectronic material, Applied Physics Letters 99 (3) (2011) 031110.

[47] K. Oe, H. Okamoto, New Semiconductor Alloy $\mathrm{GaAs}_{1-x} \mathrm{Bi}_{x}$ Grown by Metal Organic Vapor Phase Epitaxy, Japan Journal of Applied Physics 37 (Part 2, No. 11A) (1998) L1283-L1285.

[48] Y. Z. Gao, T. Yamaguchi, Liquid Phase Epitaxial Growth and Properties of InSbBi Films grown from In, Bi and Sn Solutions, Crystal Research and Technology 34 (3) (1999) 285-292. 